\title{
Out-of-Plane Free Vibration and Forced Harmonic Response of a Curved Beam
}

\author{
Hancheng Mao, ${ }^{1}$ Guangbin Yu $\left(\mathbb{D},{ }^{1,2}\right.$ Wei Liu, ${ }^{1,3}$ and Tiantian $X u^{1}$ \\ ${ }^{1}$ Key Laboratory of Advanced Manufacturing and Intelligent Technology, Ministry of Education, \\ Harbin University of Science and Technology, Harbin 150080, China \\ ${ }^{2}$ School of Mechatronics Engineering, Harbin Institute of Technology, Harbin 150001, China \\ ${ }^{3}$ School of Traffic and Transportation, Northeast Forestry University, Harbin 150040, China \\ Correspondence should be addressed to Guangbin Yu; yugbhit@hrbust.edu.cn
}

Received 13 September 2020; Revised 18 November 2020; Accepted 15 December 2020; Published 30 December 2020

Academic Editor: Mohammad Rafiee

Copyright (c) 2020 Hancheng Mao et al. This is an open access article distributed under the Creative Commons Attribution License, which permits unrestricted use, distribution, and reproduction in any medium, provided the original work is properly cited.

\begin{abstract}
Based on the governing differential equation of out-of-plane curved beam, the wave propagation behavior, free vibration, and transmission properties are presented theoretically in this paper. Firstly, harmonic wave solutions are given to investigate the dispersion relation between frequency and wave number, cut-off frequency, displacement, amplitude ratio, and phase diagram. The frequency spectrum results are obtained to verify the work by Kang and Lee. Furthermore, natural frequencies of the single and composite curved beam are calculated through solving the characteristic equation in the case of free-free, clamped-clamped, and free-clamped boundaries. Finally, the transfer matrices of the out-of-plane curved beam are derived by combining the continuity between the different interfaces. The transmissibility curves of the single and composite curved beam are compared to find the vibration attention band. This work will be valuable to extend the study of the out-of-plane vibration of curved beams.
\end{abstract}

\section{Introduction}

Curved beams are widely used in many built-up structures and arch structures because of their valuable engineering applications. Compared with straight beams, plate, and shafts, the curved beams are extremely complex, and the vibration problems of these curved beam structures are still serious, especially the out-of-plane vibration.

The free vibration and transmission response of curved members have been deeply investigated. Mace has made a detail research on the vibration behavior of beam systems by dividing waves into positive-going and negative-going waves using wave approach. He also deduced the reflection matrix in the case of simply supported, clamped, and free boundaries. From the wave point, the characteristic equation of the beam is obtained theoretically for the analysis of free vibration [1]. Huang investigated the free vibration of rotating thin rings using the wave approach. The harmonic wave solutions, frequency spectra, displacement amplitude ratio, and cut-off frequencies are also analyzed theoretically [2].

Using the governing equation, Kang analyzed the free vibration of a planar circular curved beam system through considering multiple point discontinuities such as elastic support, attached mass, and curvature changes. The dispersion equations and cut-off frequencies are determined [3]. Based on Flugge's theory, Lee considered the wave motion of in-plane curved beams with constant curvature. The displacement propagation matrix reflection matrix is derived numerically. The free vibration and energy flow associated with waves in the curved beam is also discussed in detail $[4,5]$. Howson presented the exact out-of-plane natural frequencies of the composed curved Timoshenko beams. The natural frequencies of a continuous curved beam are calculated when the effects of shear deflection and rotary inertia are considered [6]. Liu presented the radial vibration of the circular plate using the wave propagation approach and the classical method containing Bessel solution and 
Hankel solution for calculating the natural frequency theoretically [7]. Based on the in-plane and out-of-plane governing equation of the curved beam, Liu analyzed the transmission response of periodic curved beams by using the transfer matrix method and Bloch theorem. Their theoretical calculations are also simulated by FEM method [8]. Lee decoupled the governing characteristic differential equations for out-of-plane vibrations of curved nonuniform beams of constant radius, and the influence of taper ratio, center angle, and arc length on the natural frequencies of the curved beams is illustrated [9]. Tufekci investigated the out-of-plane free vibration of a circular arch with uniform cross-section by taking into account the effects of transverse shear and rotatory inertia. The results showed that flexural and torsional rotatory inertia and shear deformation have essential effects on resonance frequency, even if slender shallow arches are considered [10]. Wu proposed a new approach for free vibration analysis of arches with the effects of shear deformation and rotary inertia considered. He calculated the natural frequencies for the clamped-clamped and free-free boundaries [11]. To obtain the natural frequencies and mode shapes of curved beams with hinged-hinged, hinged-clamped, and clamped-clamped boundaries, Lee derived the differential equations governing out-of-plane free vibrations of the elastic curved beams with variable curvature. Their experimental measures show that the natural frequencies are in good agreement with the theoretical results [12]. Through adopting the classical Kirchhoff thin plate theory, Zadeh summarizes the effects of dispersion relation curves on the out-of-plane wave propagation in planar elastic plates with constant curvature [13]. Walsh studied the vibration power transmission in curved beams, considering the moment of inertia and shear deformation [14]. Chidambaram summarized the extensive published literature on the vibrations of curved bars, beams, rings, and arches of arbitrary shape. He deduced the characteristic differential equations of the in-plane, out-of-plane, and coupled vibrations theoretically. His researches mainly focus on the natural frequencies for the extensional modes and inextensional modes with different boundary conditions [15]. Wang investigated the inplane vibration of a curved beam by considering the moment of inertia and shear effect. He obtained the dispersion relation of wave number and frequency and analyzed the radial and tangential coupled band gaps of a periodic curved beam [16].

The above cited literatures mainly focus on in-plane vibration analysis of curved beams, while the out-of-plane vibration analysis of curved beams is rare. Additionally, to the authors' knowledge, lots of out-of-plane free vibration and transmission response of curved members are investigated in the case of not considering the inertia moment and shear force. To accurately show the dynamic behavior, this paper attempts to make a detailed investigation of curved beams including two factors for analyzing the dispersion relation, free vibration, and transmission response.

The paper is organized into five sections. Section 1 is a brief introduction. In Section 2, the characteristic equation of the out-of-plane vibration for the curved beam is calculated theoretically by considering the moment of inertia and shear effect. In Section 3, the natural frequencies of single and composite curved beam models with clampedclamped, free-free, and clamped-free boundaries are calculated using the classical method. In Section 4, the transmission response of the single and periodic curved beam model is obtained. Section 5 is the conclusion.

\section{Theoretical Analysis}

2.1. Governing Equations. Consider a small segment of an out-of-plane curved beam model, shown in Figure 1, where $w$ is the transverse displacement, $\psi$ is the bending slope, $\phi$ is the rotation angle, $S$ is the torsional moment, $M$ is the bending moment about a radial axis, and $Q$ is the shear force. After considering the shear force and inertia moment, the coupled governing equation of out-of-plane curved beam can be written as follows [6]:

$$
\begin{array}{r}
\frac{1}{R} \frac{\partial Q}{\partial \theta}=\rho A \frac{\partial^{2} w}{\partial t^{2}} \\
\frac{1}{R} \frac{\partial M}{\partial \theta}+\frac{S}{R}-Q=\rho I \frac{\partial^{2} \psi}{\partial t^{2}} \\
\frac{1}{R} \frac{\partial S}{\partial \theta}-\frac{M}{R}=\rho I \frac{\partial^{2} \phi}{\partial t^{2}}
\end{array}
$$

where $\rho$ denotes the density, $A$ the cross-sectional area, $I$ the second moment of area, $I_{p}$ the polar second moment, and $R$ the radius of the center.

2.2. Harmonic Wave Solution. To solve equations (1)-(3), $w$, $\psi$, and $\phi$ are given by a harmonic form:

$$
\begin{aligned}
& w(\theta, t)=W(\theta) e^{j \omega t}, \\
& \psi(\theta, t)=\Psi(\theta) e^{j \omega t}, \\
& \phi(\theta, t)=\Phi(\theta) e^{j \omega t},
\end{aligned}
$$

where $\omega$ denotes the circle frequency, $j=\sqrt{-1}$ is the imaginary unit, $W(\theta), \Psi(\theta)$, and $\Phi(\theta)$ are the amplitude of the transverse displacement, bending slope, and rotation angle, respectively. Submitting equations (4)-(6) into equations (1)-(3), one can have

$$
\begin{aligned}
& \frac{\kappa A G}{R^{2}} \frac{\mathrm{d}^{2} W}{\mathrm{~d} \theta^{2}}+\frac{\kappa A G}{R} \frac{\mathrm{d} \Psi}{\mathrm{d} \theta}=-\omega^{2} \rho A W, \\
& -\frac{\kappa A G}{R} \frac{\mathrm{d} W}{\mathrm{~d} \theta}+\frac{E I+G J}{R^{2}} \frac{\mathrm{d} \Phi}{\mathrm{d} \theta}+\frac{E I}{R^{2}} \frac{\mathrm{d}^{2} \Psi}{\mathrm{d} \theta^{2}} \\
& -\left(\frac{G J}{R^{2}}+\kappa A G\right) \Psi=-\omega^{2} \rho I \Psi, \\
& \frac{G J}{R^{2}} \frac{\mathrm{d}^{2} \Phi}{\mathrm{d} \theta^{2}}-\frac{E I}{R^{2}} \Phi-\frac{E I+G J}{R^{2}} \frac{\mathrm{d} \Psi}{\mathrm{d} \theta}=-\omega^{2} \rho I_{p} \Phi .
\end{aligned}
$$




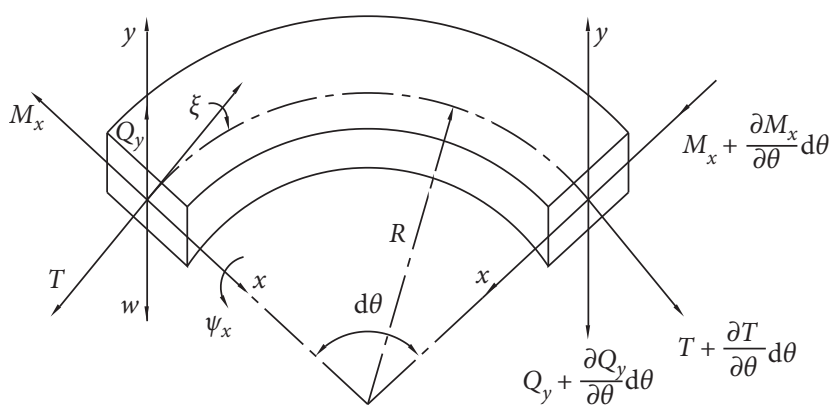

FIGURE 1: Schematic of out-of-plane vibration of a curved beam.

Equations (7)-(9) can be arranged as the following matrix form:

$$
\begin{gathered}
{\left[\begin{array}{cc}
\frac{\kappa A G}{R^{2}} D^{2}+\omega^{2} \rho A & \frac{\kappa A G}{R} D \\
-\frac{\kappa A G}{R} D & \frac{E I}{R^{2}} D^{2}-\left(\frac{G J}{R^{2}}+\kappa A G\right) \\
0 & -\frac{E I+G J}{R^{2}} D
\end{array}\right.} \\
\text { king the determinant of the coefficient } \\
\left.+\mu\left(r^{2}+s^{2}\right) \gamma^{2} \lambda^{2}\right] \beta^{4}+\left[\mu\left(1-\gamma^{2}\left(\eta+\lambda^{2}\right)\right)\right. \\
\left.\left.-\mu s^{2} \gamma^{2} \lambda^{2}\right) \gamma^{2} \lambda^{2}+s^{2}\left(\eta \gamma^{2}+2 \mu\right) \gamma^{2} \lambda^{2}\right] \beta^{2} \\
\left.\left(1-\eta \gamma^{2}\right)+r^{2} s^{2}\left(\eta \gamma^{2}-1\right) \gamma^{2} \lambda^{2}\right] \gamma^{2} \lambda^{2}=0 .
\end{gathered}
$$

Here, the specific expression of parameters gives as follows:

$$
\begin{aligned}
D & =\frac{\mathrm{d}}{\mathrm{d} \theta}, \\
r^{2} & =\frac{I}{A R^{2}}, \\
s^{2} \kappa R^{2} & =E I, \\
\kappa & =k^{\prime} A G, \\
\mu E I & =G J, \\
\eta I & =I_{p}, \\
\lambda^{2} I & =A R^{2},
\end{aligned}
$$

$$
\gamma^{2} E=\rho \omega^{2} R^{2} .
$$

According to the definition of harmonic motion, one can obtain the following relations $W, \Psi, \Phi, S, M$, and $Q$ :

$$
\begin{aligned}
& W(\theta)=\sum_{j=1}^{6} H_{1 i} C_{i} e^{\beta_{i} \theta}, \\
& \Psi(\theta)=\sum_{j=1}^{6} H_{2 i} C_{i} e^{\beta_{i} \theta}, \\
& \Phi(\theta)=\sum_{j=1}^{6} H_{3 i} C_{i} e^{\beta_{i} \theta}, \\
& S(\theta)=\sum_{j=1}^{6} H_{4 i} C_{i} e^{\beta_{i} \theta} \\
& M(\theta)=\sum_{j=1}^{6} H_{5 i} C_{i} e^{\beta_{i} \theta}, \\
& Q(\theta)=\sum_{j=1}^{6} H_{6 i} C_{i} e^{\beta_{i} \theta} .
\end{aligned}
$$

$\beta_{i}(i=1,2,3,4,5,6)$ then can be determined as the root of the characteristic equations (2)-(19), and $g=\beta^{2}$. Thus, $g$ satisfies the following relation: 


$$
\begin{gathered}
\mu g^{3}+\left[2 \mu+\eta \gamma^{2}+\mu\left(r^{2}+s^{2}\right) \gamma^{2} \lambda^{2}\right] g^{2}+\left[\mu\left(1-\gamma^{2}\left(\eta+\lambda^{2}\right)\right)\right. \\
\left.-r^{2}\left(1-\eta \gamma^{2}-\mu s^{2} \gamma^{2} \lambda^{2}\right) \gamma^{2} \lambda^{2}+s^{2}\left(\eta \gamma^{2}+2 \mu\right) \gamma^{2} \lambda^{2}\right] g \\
+\left[\left(1+\mu s^{2}\right)\left(1-\eta \gamma^{2}\right)+r^{2} s^{2}\left(\eta \gamma^{2}-1\right) \gamma^{2} \lambda^{2}\right] \gamma^{2} \lambda^{2}=0
\end{gathered}
$$

where $g_{1}, g_{2}$, and $g_{3}$ are the roots of equation (20). Therefore, $\beta$ can be rewritten as follows:

$$
\begin{aligned}
& \beta_{1}=-\beta_{4}=\sqrt{g_{1}}, \\
& \beta_{2}=-\beta_{5}=\sqrt{g_{2}}, \\
& \beta_{3}=-\beta_{6}=\sqrt{g_{3}} .
\end{aligned}
$$

To simplify conveniently, here $H_{3 i}=1$, the following relations can be obtained:

$$
\begin{aligned}
& H_{1 i}=\frac{W(\theta)}{\Phi(\theta)}=\frac{H_{1 i}}{H_{3 i}}=\frac{(1+\mu)\left(\beta_{i}^{2}+s^{2} \gamma^{2} \lambda^{2}\right)}{R\left(1-\mu \beta_{i}^{2}-\eta \gamma^{2}\right)}, \\
& H_{2 i}=\Psi(\theta)=\frac{\beta_{i}\left(1+s^{2} \beta_{i}^{2}+s^{4} \gamma^{2} \lambda^{2}\right)-R s^{2} \beta_{i}(1+\mu) H_{1 i}}{R\left[s^{2}\left(r^{2} \gamma^{2} \lambda^{2}-\mu\right)-1\right]} \\
& H_{4 i}=S(\theta)=\frac{E I_{x} \mu\left(\beta_{i} H_{1 i}-H_{2 i}\right)}{R}, \\
& H_{5 i}=M(\theta)=E I_{x}\left(\beta_{i} H_{2 i}+H_{1 i}\right), \\
& H_{6 i}=Q(\theta)=\frac{E I_{x} \beta_{i}\left[(1+\mu) R H_{1 i}-\beta_{i}^{2}+\mu-\left(r^{2}+s^{2}\right) \gamma^{2} \lambda^{2}\right]}{R^{3}\left[1+s^{2}\left(\mu-r^{2} \gamma^{2} \lambda^{2}\right)\right]} .
\end{aligned}
$$

2.2.1. Cut-Off Frequency. Equation (11) describes the dispersion frequency spectrum relations for the wave propagation in the curved beam. The cut-off frequency, denoted by $f_{c}=\omega_{c} /(2 \pi)$, defines this additional frequency spectrum for the curved beam. When the limit of $\beta$ tends to zero, equation (11) can be further simplified as

$$
\lim _{\beta \longrightarrow 0}(14)=\left[\left(1+\mu s^{2}\right)\left(1-\eta \gamma^{2}\right)+r^{2} s^{2}\left(\eta \gamma^{2}-1\right) \gamma^{2} \lambda^{2}\right] \gamma^{2} \lambda^{2}=0 .
$$

Through solving equation (27), the three roots for $\gamma$ are given by

$$
\begin{aligned}
& \gamma_{1}=0, \\
& \gamma_{2}=\frac{1}{\sqrt{\eta}}, \\
& \gamma_{3}=\frac{\sqrt{1+\mu s^{2}}}{\lambda r s} .
\end{aligned}
$$

From equation (13d), one can have

$$
\omega_{c}=\frac{\gamma}{R} \sqrt{\frac{E}{\rho}} .
$$

Here, $\gamma$ gives the following three roots:

(i) Considering the first root $\gamma_{1}=0$, namely, $\omega_{c}=0$, from equations (29) and (30), obtain $W(\theta)=0$, $\Psi(\theta)=0$, and $\Phi(\theta) \neq 0$. The abovementioned characteristics reveal that there is only rotation motion, but no bending motion and no torsional motion.

(ii) Considering the second root $\gamma_{2}=1 / \sqrt{\eta}$, namely, $\omega_{c}=(1 / R) \sqrt{(E / \rho \eta)}$, from equations (29) and (30), obtain $W(\theta) \neq 0, \Psi(\theta) \neq 0$, and $\Phi(\theta)=0$. It reveals that the curved beam only has bending motion and torsional motion, but no rotation motion.

(iii) Consider the third root $\gamma_{3}=\left(\sqrt{1+\mu s^{2}}\right) / \lambda r s$, namely, $\omega_{c}=(1 / \lambda r s R) \sqrt{\left(E\left(1+\mu s^{2}\right)\right) / \rho}$. According to equations (29) and (30), it indicates $W(\theta) \neq 0, \Psi(\theta)=0$, and $\Phi(\theta) \neq 0$, resulting in only bending motion and rotation motion, but no torsional motion.

2.2.2. Frequency Spectrum. Waves can be divided into positive-going waves and negative-going wave while the wave propagates in the curved beam. These wave motion behaviors are essential for the characteristics of the curved beam. Here, the material parameter is given in Table 1, and the structural parameter is selected as follows.

Structural parameter: radius $R=0.075 \mathrm{~m}$, length $b_{1}=0.012 \mathrm{~m}$, width $b_{2}=0.012 \mathrm{~m}$, thickness $h=0.012 \mathrm{~m}$, and shape factor $\kappa=6 / 5$.

Through calculating the roots of characteristic equation (20), the dispersion relations of the curved beam can be obtained theoretically. Shown in Figure 2 are the real wavenumber and imaginary wavenumber motion behaviors of the frequency spectrum for the curved beam model.

Figure 2 indicates that these waves divide the frequency spectrum into four regions while the wave propagates in this model, namely, region I, region II, region III, and region IV. There are three bifurcation points $f_{a}, f_{b}$, and $f_{c}$. It can then be observed that these bifurcation points play an important role in determining the wave motion behavior. Here, the value of cut-off frequency can be calculated as $f_{c}=(\gamma \sqrt{E / \rho}) / 2 \pi R=2605 \mathrm{~Hz}$, depicted in Figure 2. At this frequency, the circumference of the curved beam happens to hold a radial wavelength, and it is called the ring frequency [4].

Equation (20) can be considered as a cubic equation containing a variable parameter $g$. Due to $g=\beta^{2}$, equation (20) has six roots, namely, three forward propagating waves and three negative propagating waves. As is indicated in Figure 2, the frequency spectra are symmetrical for the extensional curved beam model [2]. In region I $0<f<f_{a}$, the wave modes are all pure real so that the overall wave can propagate along the curved beam without attenuation. In region II $f_{b}>f>f_{a}$, due to the complex wave, there are real and imagined coupled wave modes, which indicate the waves are positive-going and negative-going. In region III $f_{c}>f>f_{b}$, it occurs in the similar cases that the waves are both positive-going and negative-going. In region IV $f>f_{c}$, 
TABLE 1: Material parameters.

\begin{tabular}{lccc}
\hline Material & Density, $\rho\left(\mathrm{kg} / \mathrm{m}^{3}\right)$ & Young's modulus, $E(\mathrm{~Pa})$ & Shear modulus, $G(\mathrm{~Pa})$ \\
\hline A(PMMA) & 1062 & $0.32 \times 10^{10}$ & $0.12 \times 10^{10}$ \\
\hline
\end{tabular}

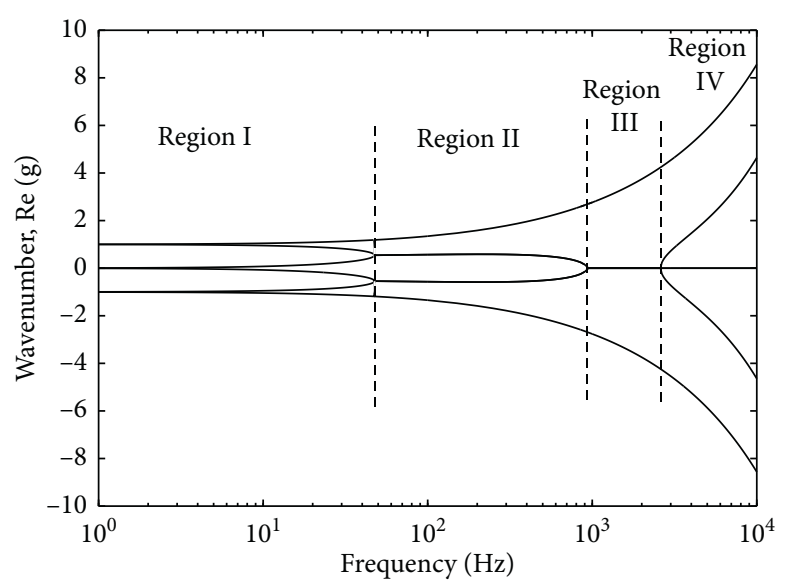

(a)

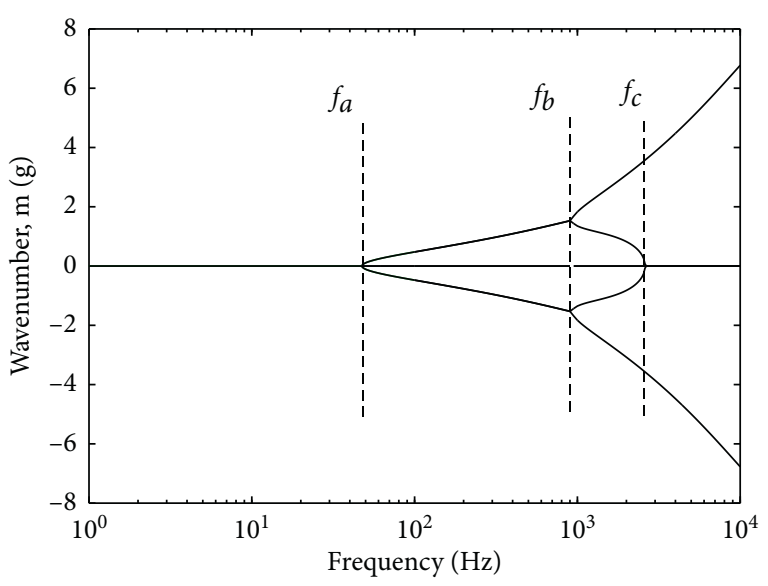

(b)

FIGURE 2: Dispersion relations of out-of-plane vibration of a curved beam. (a) Real wavenumber values. (b) Imaginary wavenumber values.

only one wave mode is purely real, the corresponding propagating wave, and the other wave will be dissipated gradually.

Based on Love's theory, Kang derived the in-plane equation of the curved beam and analyzed the dispersion relations of the frequency spectrum, shown in Figure 3. Similarly, based on Flugge's theory, Lee also obtained the dispersion relations shown in Figures 4 and 5, and the present results verify the correctness of Kang's work. These works are very significant for the analysis of curved beam models, while it is a simplified model with neglecting the effects of rotary inertia shear deformation. Actually, these factors are essential and cannot be ignored. Now, the researches on out-of-plane are rare. To make a further research, this paper not only emphasizes the dispersion relations but also further focuses on the free vibration and forced response.

2.2.3. Amplitude Ratio of Displacement. The transverse displacement $w$, bending slope $\psi$, and rotation angle $\phi$ of out-of-plane vibration of the curved beam are not independent of each other. That is to say, they are coupled with each other. From equations (22) and (23), the amplitude ratio and phase of the transverse displacement and bending slope can be obtained theoretically for the three positivegoing waves through assuming $H_{31}=H_{32}=H_{33}=1$.

Figure 6 shows the amplitude ratio and phase of the transverse displacement to the rotation angle for the three positive-going waves for the curved beam. Similarly, Figure 7 shows the amplitude ratio and phase of the bending slope to the rotation angle for the three positive-going waves for the curved beam. From Figures 6 and 7, it can be seen that the four regions shown in Figure 2 are not marked apparently, but the cut-off frequency $f_{c}$ is marked. Additionally, it can be observed that the phases of the transverse displacement and bending slope are between $-\pi / 2$ and $\pi / 2$.

\section{Free Vibration of the Curved Beam}

In this section, the free vibrations of out-of-plane vibrations for the single curved beam are given theoretically by adopting the classical method. According to the classical boundary condition, the characteristic equation of the curved beam can be obtained.

3.1. For a Single Model. The natural frequencies of out-ofplane vibration for the single curved beam shown in Figure 8 are given theoretically by adopting the classical method. According to the classical boundary condition, the characteristic equation of the curved beam can be obtained.

For a curved beam with clamped-clamped boundaries, one can obtain

$$
\left\{\begin{array}{l}
W(0)=\sum_{j=1}^{6} H_{1 i} C_{i} e^{\beta_{i} * 0}=0, \\
\Psi(0)=\sum_{j=1}^{6} H_{2 i} C_{i} e^{\beta_{i} * 0}=0, \\
\Phi(0)=\sum_{j=1}^{6} H_{3 i} C_{i} e^{\beta_{i} * 0}=0, \\
W(\pi)=\sum_{j=1}^{6} H_{1 i} C_{i} e^{\beta_{i} * \pi}=0 \\
\Psi(\pi)=\sum_{j=1}^{6} H_{2 i} C_{i} e^{\beta_{i} * \pi}=0, \\
\Phi(\pi)=\sum_{j=1}^{6} H_{3 i} C_{i} e^{\beta_{i} * \pi}=0 .
\end{array}\right.
$$




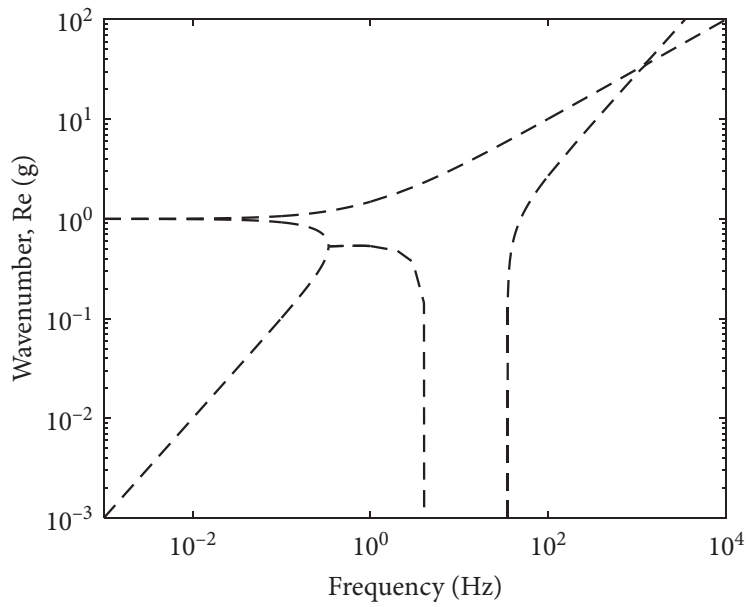

(a)

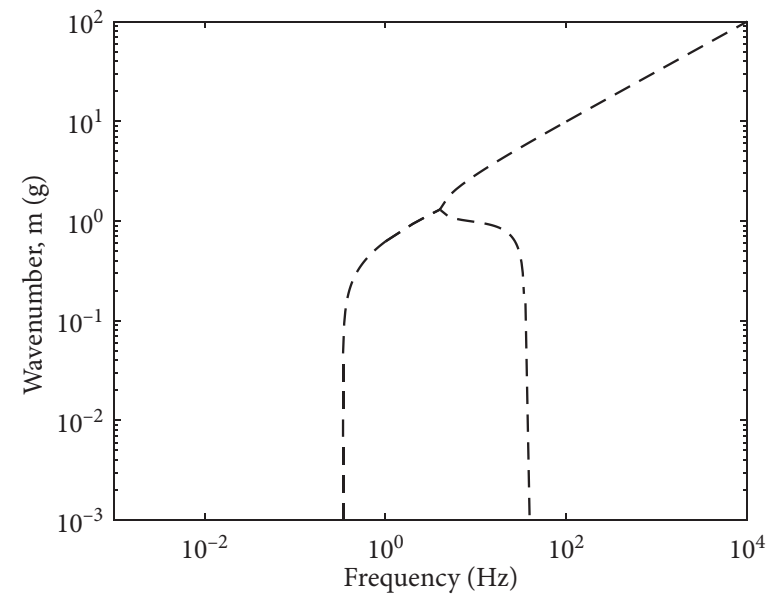

(b)

FIGURE 3: Frequency spectrum of a curved beam with $k=0.0289$. (a) Real wavenumber value. (b) Imaginary wavenumber values (extensional by Kang).

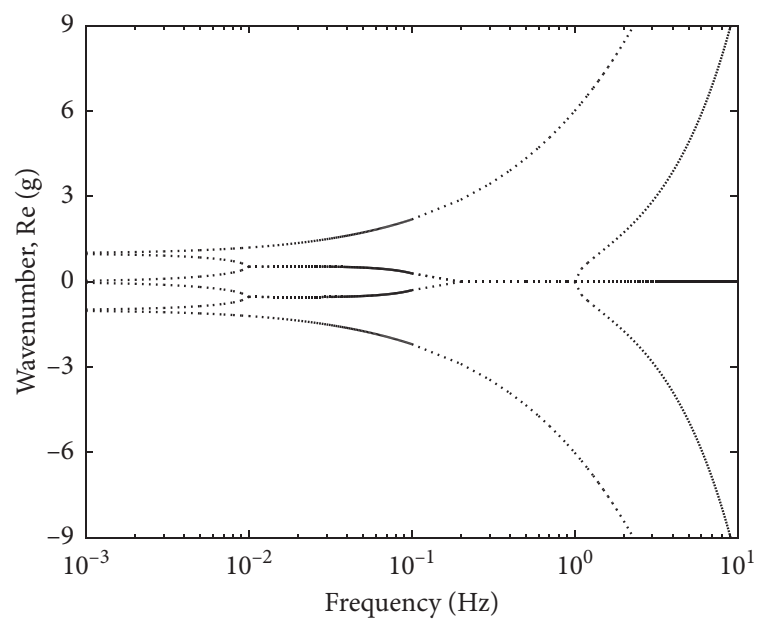

Figure 4: Frequency spectra (by Lee).

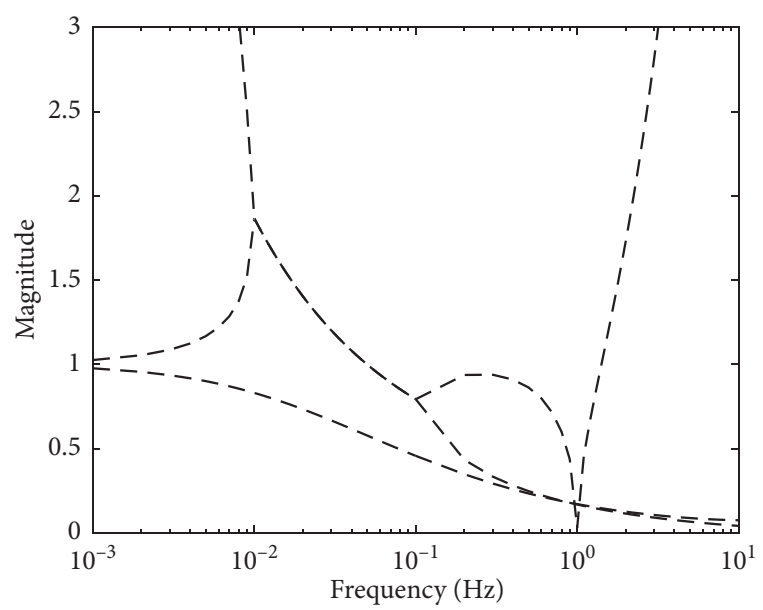

FIgURe 5: Amplitude radio (by Lee).
For the free-free boundaries, one has the following relations:

$$
\left\{\begin{array}{l}
S(0)=\sum_{j=1}^{6} H_{4 i} C_{i} e^{\beta_{i} * 0}=0 \\
M(0)=\sum_{j=1}^{6} H_{5 i} C_{i} e^{\beta_{i} * 0}=0 \\
Q(0)=\sum_{j=1}^{6} H_{6 i} C_{i} e^{\beta_{i} * 0}=0 \\
S(\pi)=\sum_{j=1}^{6} H_{4 i} C_{i} e^{\beta_{i} * \pi}=0 \\
M(\pi)=\sum_{j=1}^{6} H_{5 i} C_{i} e^{\beta_{i} * \pi}=0 \\
Q(\pi)=\sum_{j=1}^{6} H_{6 i} C_{i} e^{\beta_{i} * \pi}=0 .
\end{array}\right.
$$

For the clamped-free boundaries, it gives

$$
\left\{\begin{array}{l}
W(0)=\sum_{j=1}^{6} H_{1 i} C_{i} e^{\beta_{i} * 0}=0 \\
\Psi(0)=\sum_{j=1}^{6} H_{2 i} C_{i} e^{\beta_{i} * 0}=0 \\
\Phi(0)=\sum_{j=1}^{6} H_{3 i} C_{i} e^{\beta_{i} * 0}=0 \\
S(\pi)=\sum_{j=1}^{6} H_{4 i} C_{i} e^{\beta_{i} * \pi}=0 \\
M(\pi)=\sum_{j=1}^{6} H_{5 i} C_{i} e^{\beta_{i} * \pi}=0 \\
Q(\pi)=\sum_{j=1}^{6} H_{6 i} C_{i} e^{\beta_{i} * \pi}=0 .
\end{array}\right.
$$




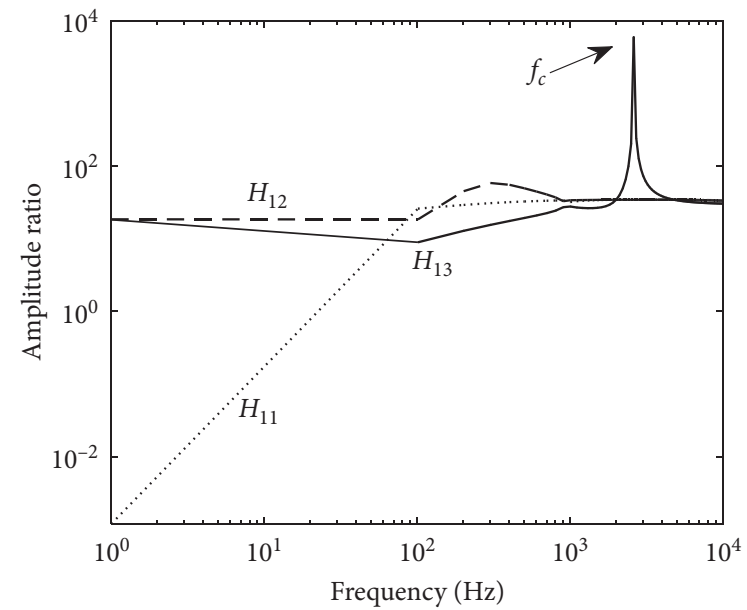

(a)

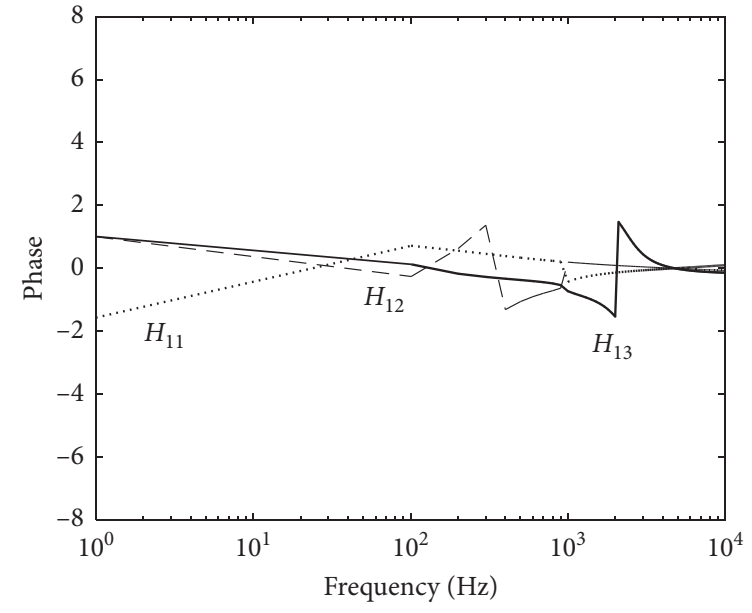

(b)

Figure 6: Amplitude ratio and phase of the transverse displacement to the rotation angle for the curved beam (a) and amplitude ratio (b) phase.

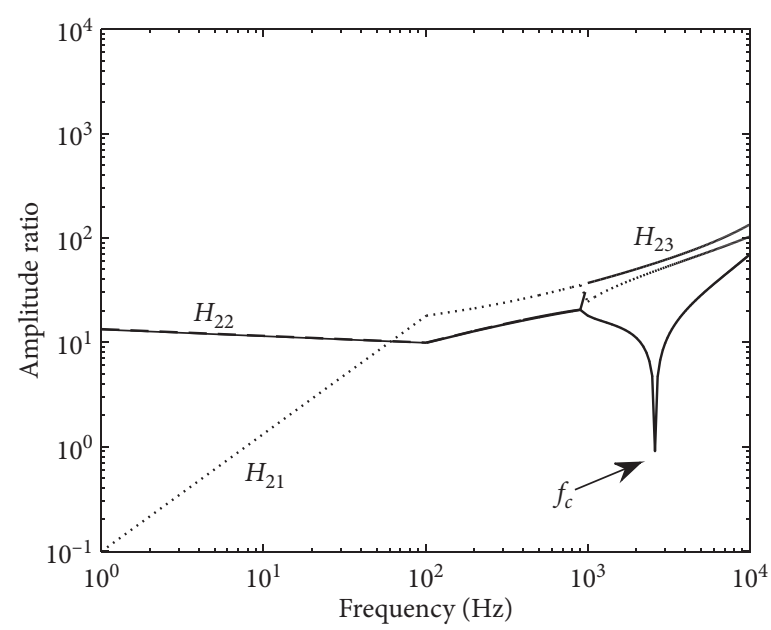

(a)

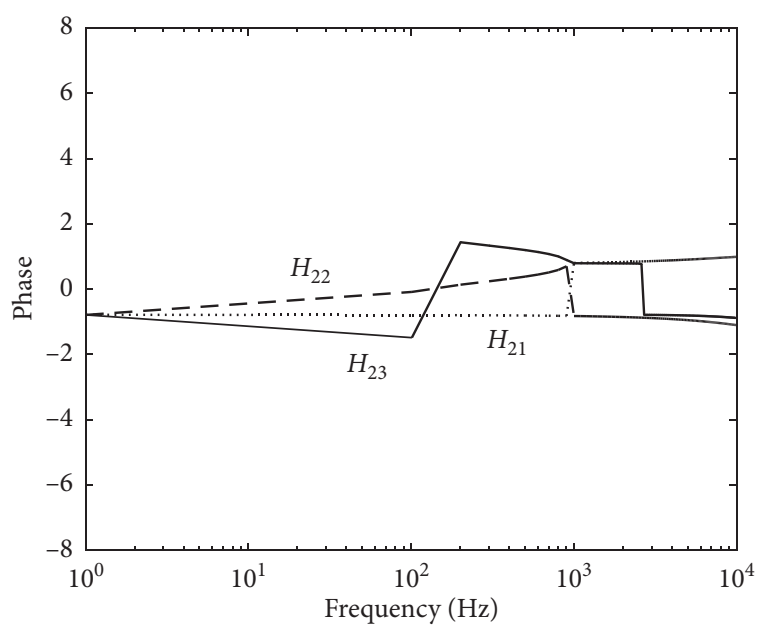

(b)

Figure 7: Amplitude ratio and phase of the bending slope to the rotation angle for the curved beam (a) and amplitude ratio (b) phase.

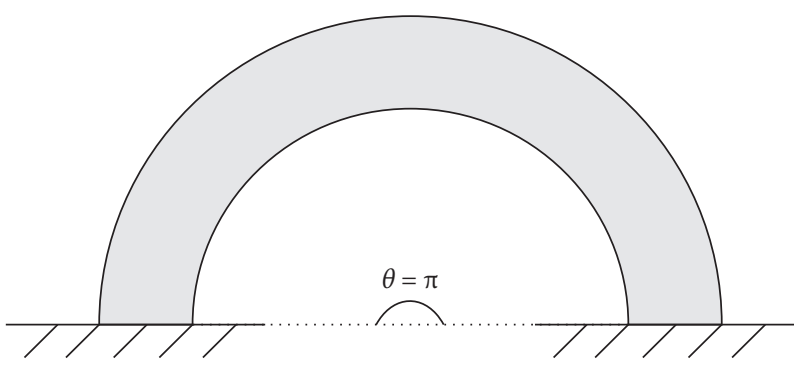

FIgURE 8: A single curved beam model. 
In this case, equation (26) can be further reduced to

$$
C(\omega)=\left[\begin{array}{c}
\sum_{j=1}^{6} H_{1 i} e^{\beta_{i} * 0} \\
\sum_{j=1}^{6} H_{2 i} e^{\beta_{i} * 0} \\
\sum_{j=1}^{6} H_{3 i} e^{\beta_{i} * 0} \\
\sum_{j=1}^{6} H_{4 i} e^{\beta_{i} * \pi} \\
\sum_{j=1}^{6} H_{5 i} e^{\beta_{i} * \pi} \\
\sum_{j=1}^{6} H_{6 i} e^{\beta_{i} * \pi}
\end{array}\right]\left\{\begin{array}{c}
C_{1} \\
C_{2} \\
C_{3} \\
C_{4} \\
C_{6}
\end{array}\right\}=0 .
$$

Equation (33) is the characteristic equation of out-ofplane vibration for the curved beam with clamped-free boundaries. Similarly, the characteristic equation with clamped-clamped and free-free boundaries also can be obtained theoretically. Figure 9 depicts the characteristic curve of natural frequency for the curved beam in the case of clamped-clamped, free-free, and clamped-free boundaries. By solving the characteristic equation, the natural frequency of the curved beam can be obtained. It can be found that the intersection point of the characteristic curve in $X$-axis is the natural frequency. Table 2 shows the natural frequencies calculated at clampedclamped and free-free boundaries. Figure 10 describes the transmission response of out-of-plane vibration for the curved beam model. It can be found that the values of natural frequency in Figure 10 are equal to the values of clamped-free case in Table 2, which illustrates the correctness of the numerical results.

3.2. For a Composite Model. Similarly, the natural frequencies of out-of-plane vibration for the composite curved beam are also calculated numerically by adopting the classical method. According to the classical boundary condition, the characteristic equation of the curved beam can be obtained.

The solving process of clamped-clamped and free-free boundaries for the composite curved beam model shown in Figure 11 are similar to the clamped-free case. Here, the clamped-free case is presented to calculate the natural frequencies. For the clamped-free boundaries, one has

$$
\left\{\begin{array}{l}
W(0)=\sum_{j=1}^{6} H_{4 i} C_{i} e^{\beta_{i} * 0}=0, \\
\Psi(0)=\sum_{j=1}^{6} H_{5 i} C_{i} e^{\beta_{i} * 0}=0, \\
\Phi(0)=\sum_{j=1}^{6} H_{6 i} C_{i} e^{\beta_{i} * 0}=0, \\
S(\pi)=\sum_{j=1}^{6} H_{4 i} C_{i} e^{\beta_{i} * \pi} T_{1}=0, \\
M(\pi)=\sum_{j=1}^{6} H_{5 i} C_{i} e^{\beta_{i} * \pi} T_{1}=0, \\
Q(\pi)=\sum_{j=1}^{6} H_{6 i} C_{i} e^{\beta_{i} * \pi} T_{1}=0 .
\end{array}\right.
$$

In this case, by combining equations (32) and (33), the following set of relations can be established:

$$
C(\omega)=\left[\begin{array}{c}
\sum_{j=1}^{6} H_{1 i} e^{\beta_{i} * 0} \\
\sum_{j=1}^{6} H_{2 i} e^{\beta_{i} * 0} \\
\sum_{j=1}^{6} H_{3 i} e^{\beta_{i} * 0} \\
\sum_{j=1}^{6} H_{4 i} e^{\beta_{i} * \pi} T_{1} \\
\sum_{j=1}^{6} H_{5 i} e^{\beta_{i} * \pi} T_{1} \\
\sum_{j=1}^{6} H_{6 i} e^{\beta_{i} * \pi} T_{1}
\end{array}\right]\left\{\begin{array}{c}
C_{1} \\
C_{2} \\
C_{3} \\
C_{4} \\
C_{5} \\
C_{6}
\end{array}\right\}=0 .
$$

Through solving the root in equation (35), the natural frequencies of out-of-plane vibration for the composite curved beam can be obtained. Figure 12 gives the characteristic curves of natural frequency of out-of-plane vibration for the composite curved beam in the case of three classical boundaries. It can be found that the intersection point of the characteristic curve in $X$-axis is the natural frequency. The values of natural frequency obtained for these three cases are given in Table 3. Figure 13 gives the transmission response of out-of-plane vibration for the composite curved beam, and the natural frequencies described in Figure 13 are the same in Table 3. That is to say, the resonance points in the transmission curves obtained by impulse response are the natural frequencies. 


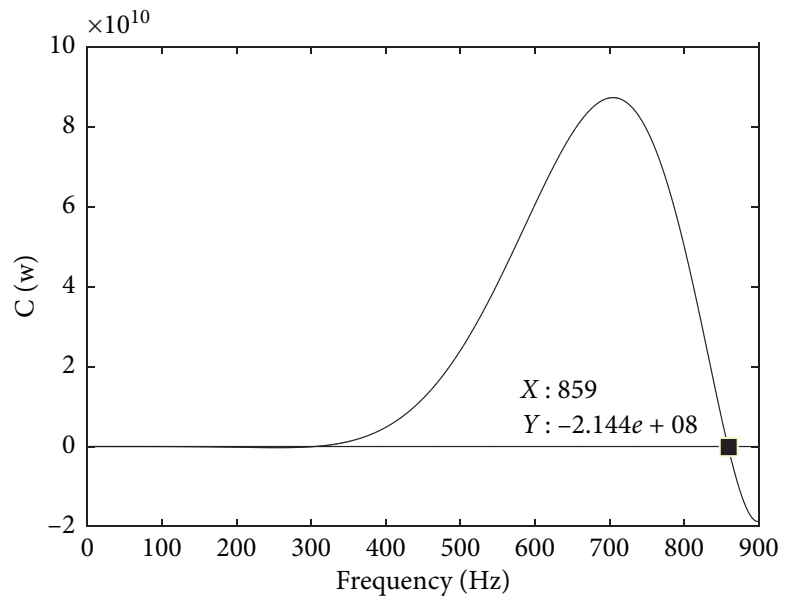

(a)

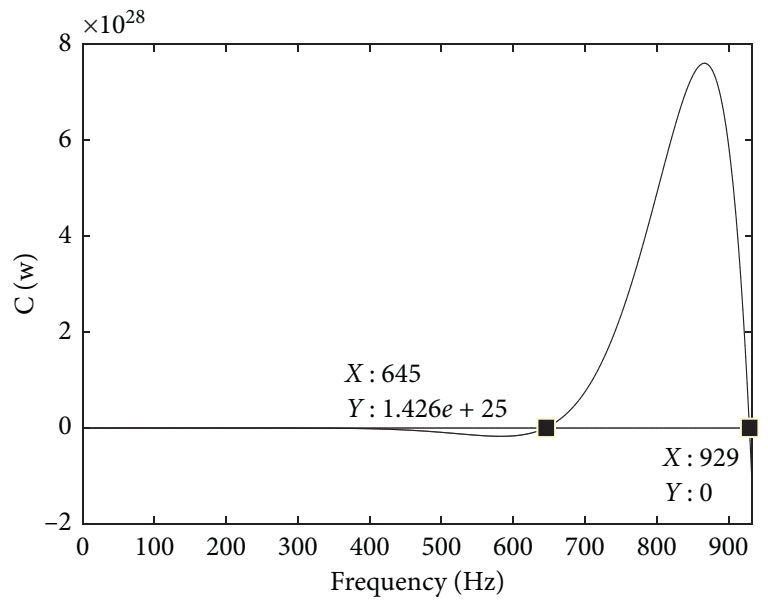

(b)

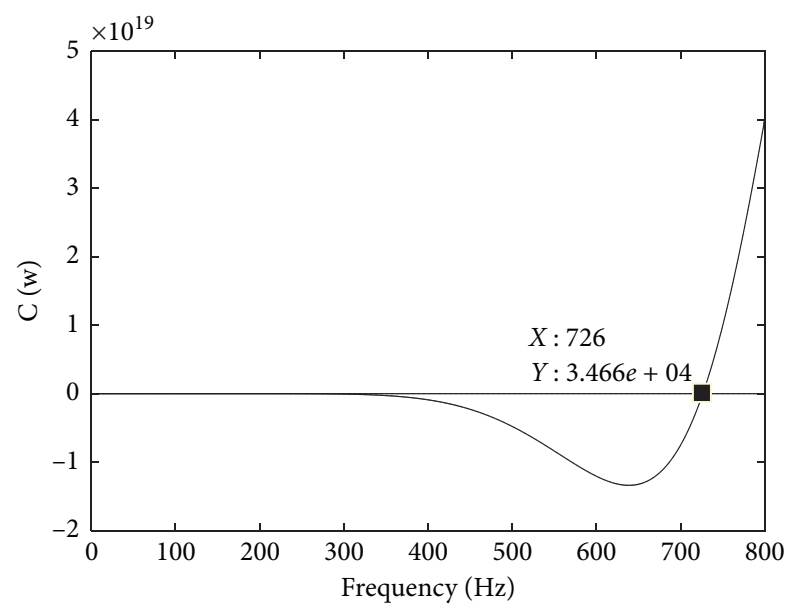

(c)

FIGURE 9: Characteristic curves of natural frequency of out-of-plane vibration for the curved beam, (a) clamped-clamped boundary, (b) freefree boundary, and (c) clamped-free boundary.

TABLE 2: Natural frequencies $(\mathrm{Hz})$ with clamped-clamped and freefree boundaries for the single model.

\begin{tabular}{lccc}
\hline Boundary & 1st mode & 2nd mode & 3rd mode \\
\hline Clamped-clamped & 47 & 305 & 859 \\
Free-free & 47 & 645 & 929 \\
Clamped-free & 64 & 218 & 726 \\
\hline
\end{tabular}

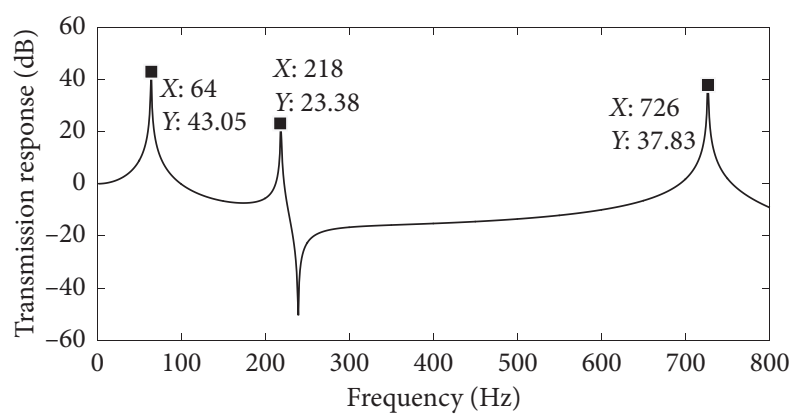

FIGURE 10: Transmission response of out-of-plane vibration for the single curved beam.

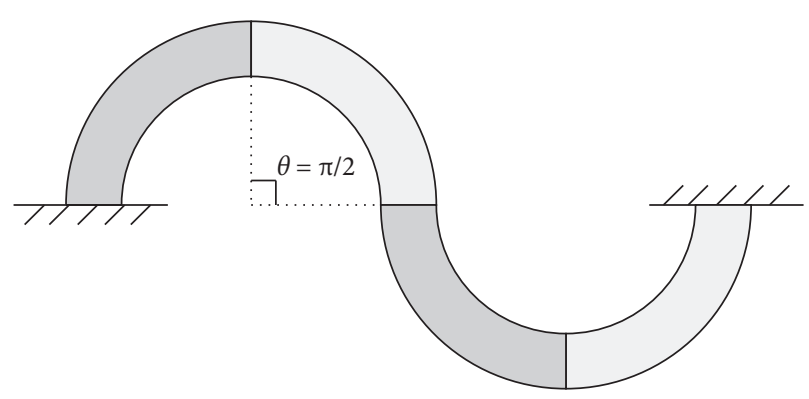

Figure 11: A composite curved beam model.

\section{Transmission Response for Periodic Curved Beam}

4.1. Base Model. In this section, to further study the transmission response, the periodic curved beam models for the out-of-plane vibration are taken as a numerical example for the analysis of the transmissibility through adopting the transfer matrix method. Herein, Figure 14 is the base model 


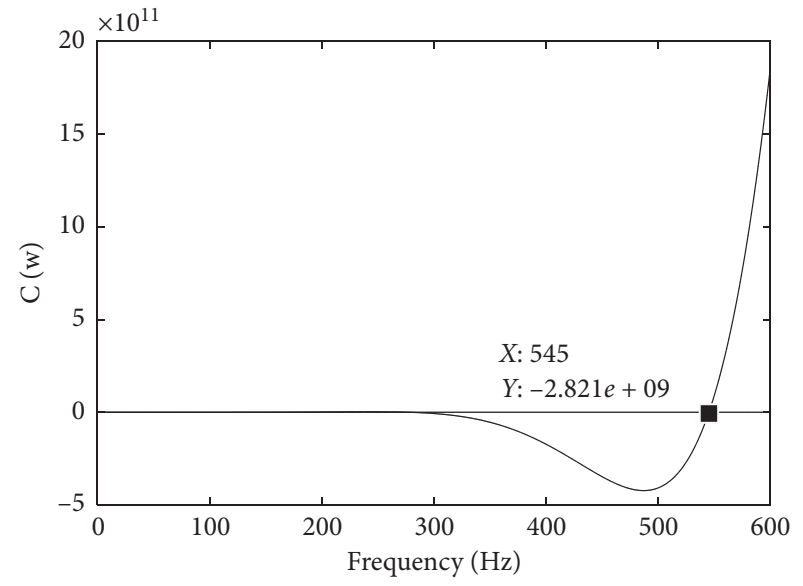

(a)

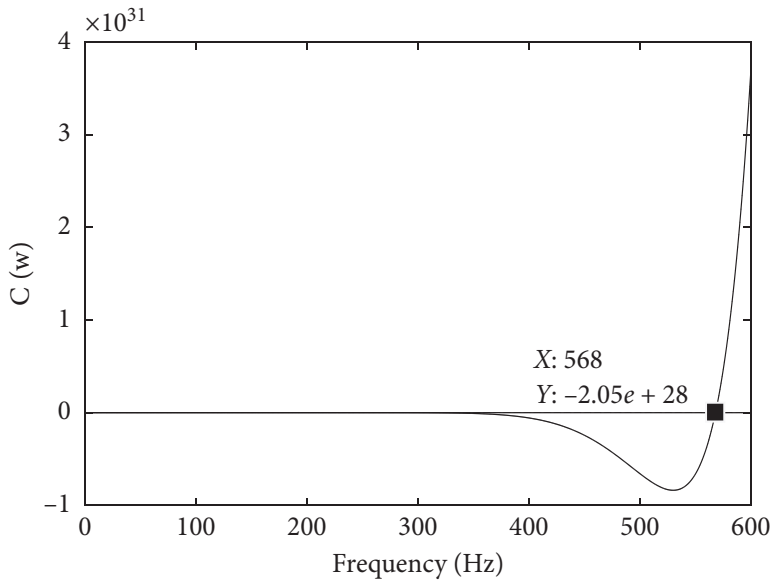

(b)

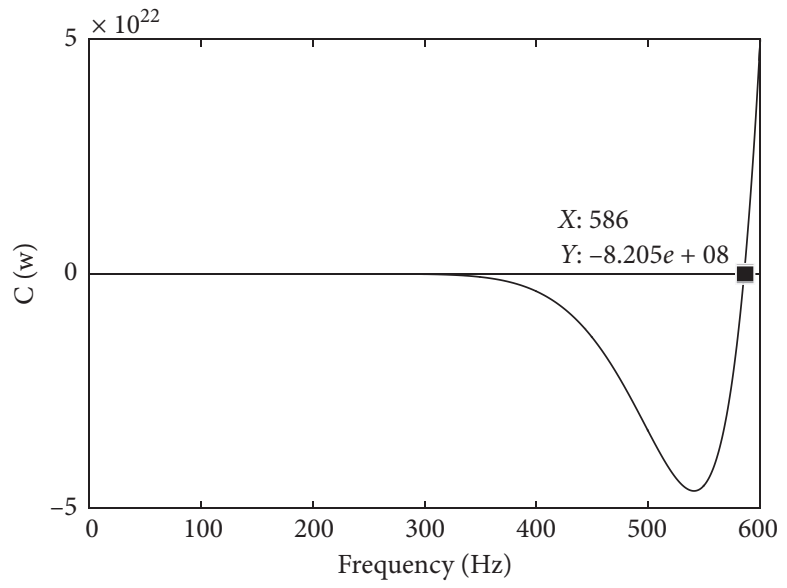

(c)

Figure 12: Characteristic curves of natural frequency of out-of-plane vibration for the curved beam, (a) clamped-clamped boundary, (b) free-free boundary, and (c) clamped-free boundary.

TABLE 3: Natural frequencies $(\mathrm{Hz})$ with clamped-clamped and free-free boundaries for the composite model.

\begin{tabular}{lcccc}
\hline Boundary & 1st mode & 2nd mode & 3rd mode & 4th mode \\
\hline Clamped-clamped & 47 & 71 & 278 & 545 \\
Free-free & 56 & 98 & 248 & 568 \\
Clamped-free & 34 & 107 & 262 & 586 \\
\hline
\end{tabular}

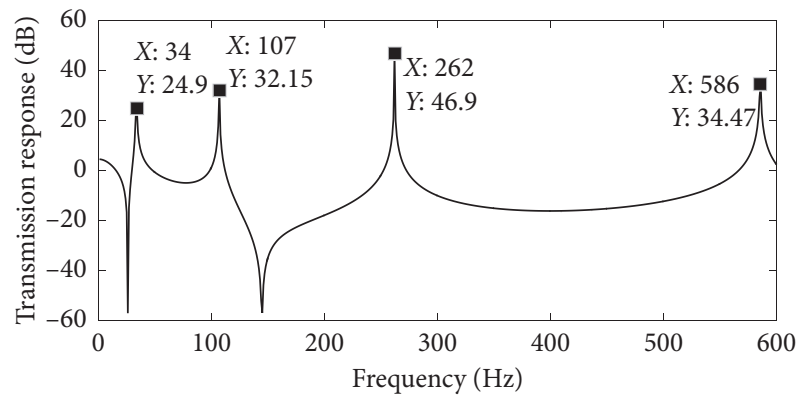

FIGURE 13: Transmission response of out-of-plane vibration for the curved beam. which is composited of eight periods, which consist of material I resin and material II steel. The angles of resin and steel are $\theta_{1}$ and $\theta_{2}$, respectively. Table 4 gives the material parameters. Shear modulus, density, and Poisson's ratio of resin are $E_{1}, \rho_{1}$, and $\delta_{1}$, respectively. Shear modulus, density, and Poisson's ratio of steel are $E_{2}, \rho_{2}$, and $\delta_{2}$.

4.2. Transfer Matrix. To obtain the transmission response of the curved beam model, the transfer matrix for the out-ofplane vibration is derived theoretically. The connection ways between the two different materials are selected with glue. Material and structural parameters have been given in 


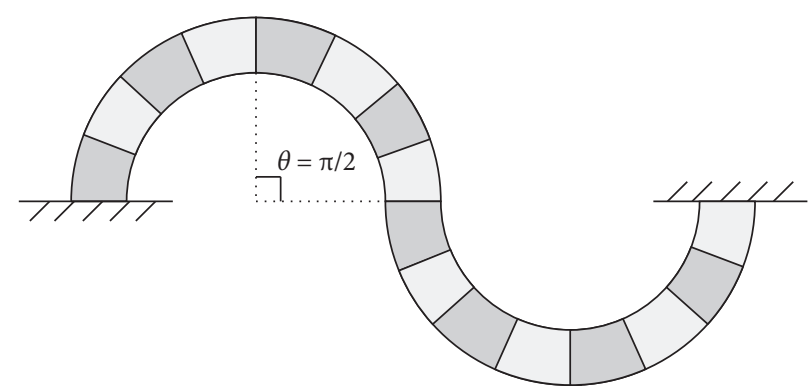

Figure 14: Eight period composite curved beam model.

TABLE 4: Material parameters.

\begin{tabular}{lccc}
\hline Material & Density, $\rho\left(\mathrm{kg} / \mathrm{m}^{3}\right)$ & Young's modulus, $E(\mathrm{~Pa})$ & Poisson's ratio \\
\hline I (PMMA) & 1062 & $0.32 \times 10^{10}$ & 0.333 \\
II (Al) & 2799 & $7.21 \times 10^{10}$ & 0.345 \\
\hline
\end{tabular}

Table 1. According to equations (14)-(19), the expression of six parameters $W, \Psi, \Phi, S, M$, and $Q$ in terms of the harmonic motion can be rewritten as follows:

$$
\begin{aligned}
& W(\theta)=H_{11} C_{1 a}^{+} e^{g_{1} \theta}+H_{12} C_{1 b}^{+} e^{g_{2} \theta}+H_{13} C_{1 c}^{+} e^{g_{3} \theta}+H_{14} C_{1 a}^{-} e^{g_{4} \theta}+H_{15} C_{1 b}^{-} e^{g_{5} \theta}+H_{16} C_{1 c}^{-} e^{g_{6} \theta}, \\
& \Psi(\theta)=H_{21} C_{1 a}^{+} e^{g_{1} \theta}+H_{22} C_{1 b}^{+} e^{g_{2} \theta}+H_{23} C_{1 c}^{+} e^{g_{3} \theta}+H_{24} C_{1 a}^{-} e^{g_{4} \theta}+H_{25} C_{1 b}^{-} e^{g_{5} \theta}+H_{26} C_{1 c}^{-} e^{g_{6} \theta}, \\
& \Phi(\theta)=H_{31} C_{1 a}^{+} e^{g_{1} \theta}+H_{32} C_{1 b}^{+} e^{g_{2} \theta}+H_{33} C_{1 c}^{+} e^{g_{3} \theta}+H_{34} C_{1 a}^{-} e^{g_{4} \theta}+H_{35} C_{1 b}^{-} e^{g_{5} \theta}+H_{36} C_{1 c}^{-} e^{g_{6} \theta}, \\
& S(\theta)=H_{41} C_{1 a}^{+} e^{g_{1} \theta}+H_{42} C_{1 b}^{+} e^{g_{2} \theta}+H_{43} C_{1 c}^{+} e^{g_{3} \theta}+H_{44} C_{1 a}^{-} e^{g_{4} \theta}+H_{45} C_{1 b}^{-} e^{g_{5} \theta}+H_{46} C_{1 c}^{-} e^{g_{6} \theta}, \\
& M(\theta)=H_{51} C_{1 a}^{+} e^{g_{1} \theta}+H_{52} C_{1 b}^{+} e^{g_{2} \theta}+H_{53} C_{1 c}^{+} e^{g_{3} \theta}+H_{54} C_{1 a}^{-} e^{g_{4} \theta}+H_{55} C_{1 b}^{-} e^{g_{5} \theta}+H_{56} C_{1 c}^{-} e^{g_{6} \theta}, \\
& Q(\theta)=H_{61} C_{1 a}^{+} e^{g_{1} \theta}+H_{62} C_{1 b}^{+} e^{g_{2} \theta}+H_{63} C_{1 c}^{+} e^{g_{3} \theta}+H_{64} C_{1 a}^{-} e^{g_{4} \theta}+H_{65} C_{1 b}^{-} e^{g_{5} \theta}+H_{66} C_{1 c}^{-} e^{g_{6} \theta} .
\end{aligned}
$$

The continuity condition on the rotation angle $\theta=\pi / 8$ between material I and material II is given by

$$
\begin{aligned}
& W_{1}\left(\frac{\pi}{6}\right)=W_{2}\left(\frac{\pi}{6}\right), \\
& \Psi_{1}\left(\frac{\pi}{6}\right)=\Psi_{2}\left(\frac{\pi}{6}\right), \\
& \Phi_{1}\left(\frac{\pi}{6}\right)=\Phi_{2}\left(\frac{\pi}{6}\right), \\
& S_{1}\left(\frac{\pi}{6}\right)=S_{2}\left(\frac{\pi}{6}\right), \\
& M_{1}\left(\frac{\pi}{6}\right)=M_{2}\left(\frac{\pi}{6}\right), \\
& Q_{1}\left(\frac{\pi}{6}\right)=Q_{2}\left(\frac{\pi}{6}\right) .
\end{aligned}
$$

The following matrix equation can be established:

$$
K_{1} \Psi_{n 2}=H_{1} \Psi_{n 1},
$$

where $\quad \Psi_{n 1}=\left[\begin{array}{llllll}P_{n 1 a}^{+} & P_{n 1 b}^{+} & P_{n 1 c}^{+} & P_{n 1 a}^{-} & P_{n 1 b}^{-} & P_{n 1 c}^{-}\end{array}\right]^{T}$ $\Psi_{n 2}=\left[\begin{array}{llllll}P_{n 2 a}^{+} & P_{n 2 b}^{+} & P_{n 2 c}^{+} & P_{n 2 a}^{-} & P_{n 2 b}^{-} & P_{n 2 c}^{-}\end{array}\right]^{T}$.
Similarly, imposing the continuity condition at $\theta_{1}=0$ and $\theta_{2}=\pi / 4$, one has

$$
\begin{aligned}
& W_{1}(0)=W_{2}\left(\frac{\pi}{3}\right), \\
& \Psi_{1}(0)=\Psi_{2}\left(\frac{\pi}{3}\right), \\
& \Phi_{1}(0)=\Phi_{2}\left(\frac{\pi}{3}\right), \\
& S_{1}(0)=S_{2}\left(\frac{\pi}{3}\right), \\
& M_{1}(0)=M_{2}\left(\frac{\pi}{3}\right), \\
& Q_{1}(0)=Q_{2}\left(\frac{\pi}{3}\right) .
\end{aligned}
$$

Then, these parameters at the continuity position lead to the following matrix relations:

$$
K_{2} \psi_{n 2}=H_{2} \psi_{(n+1) 1},
$$


where $\Psi_{(n+1) 1}=\left[P_{(n+1) 1 a}^{+} P_{(n+1) 1 b}^{+} P_{(n+1) 1 c}^{+} P_{(n+1) 1 a}^{-} P_{(n+1) 1 b}^{-} P_{(n+1) 1 c}^{-}\right]^{T}$.

Equations (38) and (40) can be combined to give

$$
\Psi_{(n+1) 1}=H_{2}^{-1} K_{2} K_{1}^{-1} H_{1} \Psi_{n 1}=T_{a} \Psi_{n 1},
$$

where $T_{a}=H_{2}^{-1} K_{2} K_{1}^{-1} H_{1}$ is the transfer matrix [13] of the periodic curved beam model.
To obtain the transmission response of this curved beam model, then suppose that the left end is used to load displacement and the right end is used to pick the displacement. Thus, the transverse displacement at the left end is considered as one unit, and the other parameters seem to be zero. Based on these definitions, the following relations can be established:

$$
\begin{aligned}
& W(0)=H_{11} C_{1 a}^{+} e^{g_{1} * 0}+H_{12} C_{1 b}^{+} e^{g_{2} * 0}+H_{13} C_{1 c}^{+} e^{g_{3} * 0}+H_{14} C_{1 a}^{-} e^{g_{4} * 0}+H_{15} C_{1 b}^{-} e^{g_{5} * 0}+H_{16} C_{1 c}^{-} e^{g_{6} * 0}=0 \\
& \Psi(0)=H_{21} C_{1 a}^{+} e^{g_{1} * 0}+H_{22} C_{1 b}^{+} e^{g_{2} * 0}+H_{23} C_{1 c}^{+} e^{g_{3} * 0}+H_{24} C_{1 a}^{-} e^{g_{4} * 0}+H_{25} C_{1 b}^{-} e^{g_{5} * 0}+H_{26} C_{1 c}^{-} e^{g_{6} * 0}=0 \\
& \Phi(0)=H_{31} C_{1 a}^{+} e^{g_{1} * 0}+H_{32} C_{1 b}^{+} e^{g_{2} * 0}+H_{33} C_{1 c}^{+} e^{g_{3} * 0}+H_{34} C_{1 a}^{-} e^{g_{4} * 0}+H_{35} C_{1 b}^{-} e^{g_{5} * 0}+H_{36} C_{1 c}^{-} e^{g_{6} * 0}=0 \\
& S\left(\frac{\pi}{4}\right)=H_{41} C_{1 a}^{+} e^{g_{1} * \frac{\pi}{4}}+H_{42} C_{1 b}^{+} e^{g_{2} * \frac{\pi}{4}}+H_{43} C_{1 c}^{+} e^{g_{3} * \frac{\pi}{4}}+H_{44} C_{1 a}^{-} e^{g_{4} * \frac{\pi}{4}}+H_{45} C_{1 b}^{-} e^{g_{5} * \frac{\pi}{4}}+H_{46} C_{1 c}^{-} e^{g_{6} * \frac{\pi}{4}}=0 \\
& M\left(\frac{\pi}{4}\right)=H_{51} C_{1 a}^{+} e^{g_{1} * \frac{\pi}{4}}+H_{52} C_{1 b}^{+} e^{g_{2} * \frac{\pi}{4}}+H_{53} C_{1 c}^{+} e^{g_{3} * \frac{\pi}{4}}+H_{54} C_{1 a}^{-} e^{g_{4} * \frac{\pi}{4}}+H_{55} C_{1 b}^{-} e^{g_{5} * \frac{\pi}{4}}+H_{56} C_{1 c}^{-} e^{g_{6} * \frac{\pi}{4}}=0 \\
& Q\left(\frac{\pi}{4}\right)=H_{61} C_{1 a}^{+} e^{g_{1} * \frac{\pi}{4}}+H_{62} C_{1 b}^{+} e^{g_{2} * \frac{\pi}{4}}+H_{63} C_{1 c}^{+} e^{g_{3} * \frac{\pi}{4}}+H_{64} C_{1 a}^{-} e^{g_{4} * \frac{\pi}{4}}+H_{65} C_{1 b}^{-} e^{g_{5} * \frac{\pi}{4}}+H_{66} C_{1 c}^{-} e^{g_{6} * \frac{\pi}{4}}=0
\end{aligned}
$$

The above equations can be rewritten into a matrix form:

$$
\left[\begin{array}{l}
C_{1 a}^{+} \\
C_{1 b}^{+} \\
C_{1 c}^{+} \\
C_{1 a}^{-} \\
C_{1 c}^{-}
\end{array}\right]=\left[\begin{array}{ccccccc}
H_{11} e^{g_{1} * 0} & H_{12} e^{g_{2} * 0} & H_{13} e^{g_{3} * 0} & H_{14} e^{g_{4} 0} & H_{15} e^{g_{5} 0} & H_{16} e^{g_{6} 0} \\
H_{21} e^{g_{1} * 0} & H_{22} e^{g_{2} * 0} & H_{23} e^{g_{3} * 0} & H_{24} e^{g_{4} 0} & H_{25} e^{g_{5} 0} & H_{26} e^{g_{6} 0} \\
H_{31} e^{g_{1} * 0} & H_{32} e^{g_{2} * 0} & H_{33} e^{g_{3} * 0} & H_{34} e^{g_{4} 0} & H_{35} e^{g_{5} 0} & H_{36} e^{g_{6} 0} \\
H_{41} e^{g_{1} * \frac{\pi}{4}} & H_{42} e^{g_{2} * \frac{\pi}{4}} & H_{43} e^{g_{3} * \frac{\pi}{4}} & H_{44} e^{g_{4} * \frac{\pi}{4}} & H_{45} e^{g_{5} * \frac{\pi}{4}} & H_{46} e^{g_{6} * \frac{\pi}{4}} \\
H_{51} e^{g_{1} * \frac{\pi}{4}} & H_{52} e^{g_{2} * \frac{\pi}{4}} & H_{53} e^{g_{3} * \frac{\pi}{4}} & H_{54} e^{g_{4} * \frac{\pi}{4}} & H_{55} e^{g_{5} * \frac{\pi}{4}} & H_{56} e^{g_{6} * \frac{\pi}{4}} \\
H_{61} e^{g_{1} * \frac{\pi}{4}} & H_{62} e^{g_{2} * \frac{\pi}{4}} & H_{63} e^{g_{3} * \frac{\pi}{4}} & H_{64} e^{g_{4} * \frac{\pi}{4}} & H_{65} e^{g_{5} * \frac{\pi}{4}} & H_{66} e^{g_{6} * \frac{\pi}{4}}
\end{array}\right]\left[\begin{array}{l}
1 \\
0 \\
0 \\
0 \\
0 \\
0 \\
0
\end{array}\right.
$$

Combining equations (46)-(48), the transverse displacements are given by

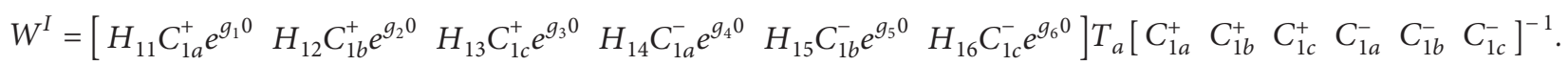


Therefore, the vibration transmission response can be defined as

$$
\mathrm{dB}=20 \log W^{I}
$$

4.3. Transmission Response. Considering the effects of inertia and shear deformation, the transmission response of the out-of-plane vibration for the curved beam model is calculated numerically by using the transfer matrix and boundary loads. The transmission response curves are plotted in Figure 14. To facilitate this investigation, the transmission responses for the case of single model and eight-periodic models are compared.

Regarding the single model, Figure 15 clearly shows that the vibration attenuation region of the transmission response is approximately $20 \mathrm{~dB}$. Actually, there is no vibration attenuation when the elastic wave propagates in a single model, such as rods, beams, and shaft. Thus, this case reveals that the vibration attenuation is significantly caused by the curvature radius of the curved beam model, and the resonance frequency is given in Table 2 .

Regarding the eight-periodic model, Figure 15 clearly indicates that there are three vibration attention regions, marked as band A $113 \mathrm{~Hz}-272 \mathrm{~Hz}$, band B $281 \mathrm{~Hz}-553 \mathrm{~Hz}$, and band $\mathrm{C} 559 \mathrm{~Hz}-800 \mathrm{~Hz}$. These low frequency bands are very significant for the vibration reduction of the engineering field, such as bridges. The resonance point in the transmission curf obtained by impulse response is the natural frequency. Thus, in many practical engineering fields, LMS Test.Lab or Pulse can be used to obtain the transmission response curves easily by adopting the hammering method for finding the natural frequency instead of testing model.

By comparing the single model and eight-periodic models, it can be observed that they have the similar vibration attention amplitude. This phenomenon indicates that the periodic model is not very efficient for the vibration attention, which is one point different from the previous conclude.

As is known for us, the curvature radius is a key factor for the curved beam and has an important influence on the transmission response. Furthermore, Figure 16 clearly gives the transmission response of the curved beam with different curvature radius. It can be seen that the region of the vibration reduction moves towards the low frequency with the curvature radius increasing, but the amplitude of vibration attention almost has no change. Thus, we can conclude that one can select a big curvature radius of the curved beam while one wants to have a low frequency band. Moreover, it should be mentioned that the attention region marked as band $D$ is $57 \mathrm{~Hz}-140 \mathrm{~Hz}$, which is very useful for avoiding resonance.

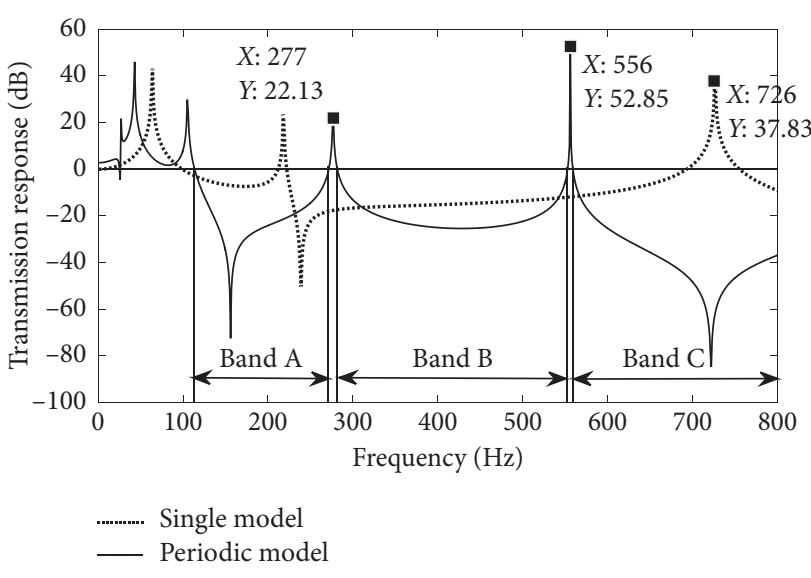

FIGURE 15: Transmission response for the curved beam with single model and eight-periodic models.

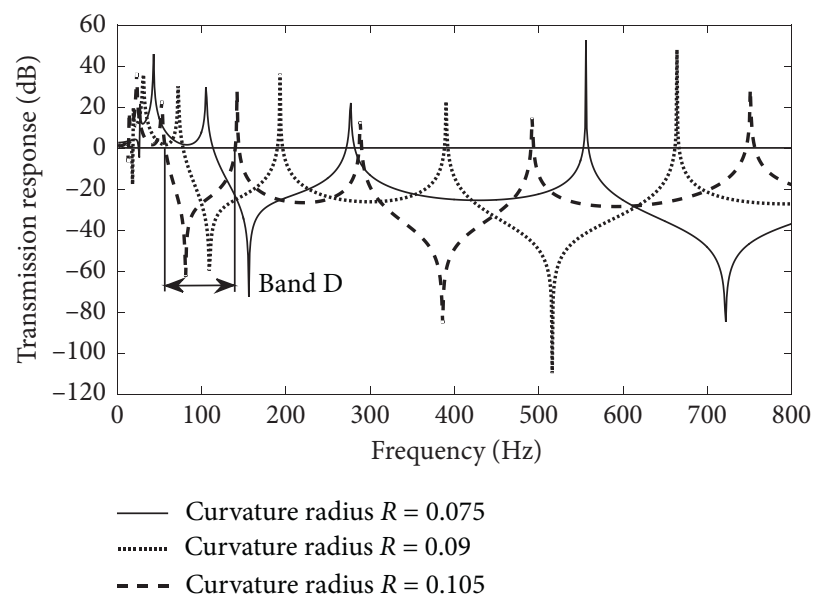

FIgURE 16: Transmission response for the curved beam with different curvature radius.

\section{Conclusion}

This paper concerns out-of-plane vibration of curved beams, considering the shear force and inertia moment. Based on the coupled governing equation of the curved beam, the dispersion relations, cut-off frequency, and displacement amplitude ratio are investigated in detail. Furthermore, the free vibration and transmission properties for the single and composite curved beam models are investigated, and the following conclusions are drawn:

(1) From the dispersion relation of the out-of-plane curved beam, it can be found that the elastic wave can be divided into three forward propagating waves and three negative propagating waves, and these waves can be divided into four regions.

(2) Natural frequencies of the single and composite curved beam model with three boundaries are 
calculated through using the harmonic wave solutions. It can be found that the resonance frequency can be obtained from the impulse response.

(3) Curvature radius is a key factor for the curved beam and has an important influence on the transmission response. It can be seen that the region of the vibration reduction moves towards the low frequency with the curvature radius increasing, but the amplitude of vibration attention almost has no change.

Our research manifests that the natural frequencies of composite TCP structures can be obtained easily using the wave approach, which can be very meaningful for many engineering fields. For example, rotating parts are often coupled with transverse vibrations (such as the gear driving systems). Apparently, analyzing the transmission properties and parameter's effect is very significant for transverse vibration suppression.

\section{Data Availability}

The raw/processed data required to reproduce these findings cannot be shared at this time as the data also form part of an ongoing study.

\section{Conflicts of Interest}

The authors declared no potential conflicts of interest with respect to the research, authorship, and/or publication of this article.

\section{Acknowledgments}

The authors wish to thank the Key Laboratory of Advanced Manufacturing and Intelligent Technology, Ministry of Education, Harbin University of Science and Technology and School of Mechatronics Engineering, Harbin Institute of Technology, for providing technical support. The research was funded by National Key Research and Development Project of China (Grant no. 2019YFB2006400) and the Major Science and Technology Projects of Heilongjiang Province (Grant no. 2019ZX03A02).

\section{References}

[1] B. R. Mace, "Wave reflection and transmission in beams," Journal of Sound and Vibration, vol. 97, no. 2, pp. 237-246, 1984.

[2] D. Huang, L. Tang, and R. Cao, "Free vibration analysis of planar rotating rings by wave propagation," Journal of Sound and Vibration, vol. 332, no. 20, pp. 4979-4997, 2013.

[3] B. Kang, C. H. Riedel, and C. A. Tan, "Free vibration analysis of planar curved beams by wave propagation," Journal of Sound and Vibration, vol. 260, no. 1, pp. 19-44, 2003.

[4] S. K. Lee, R. B. Mace, and M. J. Brennan, "Wave propagation, reflection and transmission in curved beams," Journal of Sound and Vibration, vol. 306, no. 3-5, pp. 636-656, 2007.

[5] S. K. Lee, Wave Reflection, Transmission and Propagation in Structural Waveguides, Ph.D. thesis, Southampton University, Southampton, England, UK, 2006.
[6] W. P. Howson and A. K. Jemah, "Exact out-of-plane natural frequency of curved timoshenko beams," Journal of Engineering Mechanics, vol. 125, no. 1, pp. 19-25, 2007.

[7] W. Liu, D. H. Wang, H. F. Lu, Y. M. Cao, and P. R. Zhang, "Research on radial vibration of a circular plate," Shock and Vibration, vol. 2016, Article ID 6758291, 8 pages, 2016.

[8] Y. Li, T. Chen, X. Wang, and S. Li, "Lamb wave band gaps in one-dimensional radial phononic crystal plates with periodic double-sided corrugations," Physica B: Condensed Matter, vol. 476, pp. 82-87, 2015.

[9] S. Y. Lee and J. C. Chao, "Out-of-plane vibration of curved non-uniform beams of constant radius," Journal of Sound and Vibration, vol. 238, no. 1, pp. 443-458, 2000.

[10] E. Tufekci and O. Y. Dogruer, "Out-of-plane free vibration of a circular arch with uniform cross-section: exact solution," Journal of Sound and Vibration, vol. 291, no. 3-5, pp. 525-538, 2006.

[11] J. S. Wu and L. K. Chiang, "A new approach for free vibration analysis of arches with effects of shear deformation and rotary inertia considered," Journal of Sound and Vibration, vol. 277, no. 1-2, pp. 49-71, 2004.

[12] B. K. Lee, S. J. Oh, and J. M. Mo, "Out-of-plane free vibrations of curved beams with variable curvature," Journal of Sound and Vibration, vol. 318, no. 1-2, pp. 227-246, 2008.

[13] M. N. Zadeh and S. V. Sorokin, "Simplified description of outof-plane waves in thin annular elastic plates," Journal of Sound and Vibration, vol. 332, no. 4, pp. 894-906, 2013.

[14] S. J. Walsh and R. G. White, "Vibrational power transmission in curved beams," Journal of Sound and Vibration, vol. 233, no. 3, pp. 455-488, 2000.

[15] P. Chidambaram and A. W. Leissa, "Vibration of planar curved beams, rings, and arches," Applied Mechanics Review, vol. 46, no. 9, pp. 467-483, 1993.

[16] D. Wang, S. Zhijun, L. Wei, C. Meilong, L. Siyuan, and L. Shidan, "In-plane vibration analysis of phononic crystal curved beams," Noise Control Engineering Journal, vol. 64, no. 5, pp. 658-667, 2016. 